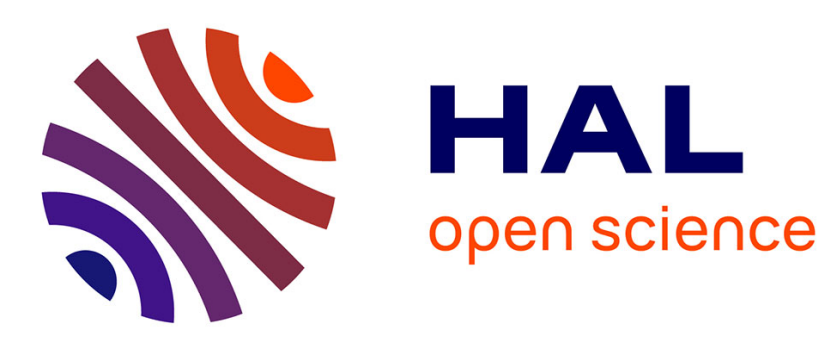

\title{
Optique de couplage encerclant
}

\author{
J. Perdijon
}

\section{To cite this version:}

J. Perdijon. Optique de couplage encerclant. Revue de Physique Appliquée, 1983, 18 (2), pp.129-133. 10.1051/rphysap:01983001802012900 . jpa-00245072

\section{HAL Id: jpa-00245072 https://hal.science/jpa-00245072}

Submitted on 1 Jan 1983

HAL is a multi-disciplinary open access archive for the deposit and dissemination of scientific research documents, whether they are published or not. The documents may come from teaching and research institutions in France or abroad, or from public or private research centers.
L'archive ouverte pluridisciplinaire HAL, est destinée au dépôt et à la diffusion de documents scientifiques de niveau recherche, publiés ou non, émanant des établissements d'enseignement et de recherche français ou étrangers, des laboratoires publics ou privés. 
Classification

Physics Abstracts

$42.60-42.80-43.35$

\title{
Optique de couplage encerclant
}

\author{
J. Perdijon \\ 771, ch. de Rosat, 38330 Saint-Ismier, France \\ (Reçu le 5 avril 1982, révisé le 17 août, accepté le 3 novembre 1982)
}

\begin{abstract}
Résumé. - Nous proposons une nouvelle famille d'optiques permettant de coupler des ondes cohérentes avec un résonateur annulaire. Ces optiques sont calculées de façon à obtenir la concordance de phase entre les ondes incidentes et les ondes guidées en tout point du guide et sur $360^{\circ}$. Nous donnons quelques exemples pour une source plane ou cylindrique, et pour un guide tubulaire ou torique.
\end{abstract}

Abstract. - We propose a new family of optics for coupling coherent waves with a ring resonator. These optics are calculated to phase incident waves and guided waves at any point on the guide and all around. We give some examples for a plane or a cylindrical source, and for a tubular or a toric guide.

1. Introduction - Les guides d'onde annulaires sont utilisés dans de nombreux dispositifs tels que résonateurs, oscillateurs ou interféromètres; ils présentent l'avantage de fonctionner avec des ondes progressives ou stationnaires. Nous ne nous intéresserons ici qu'aux guides possédant un axe de révolution; il peut s'agir par exemple d'une cavité torique dans laquelle circulent des micro-ondes [1], ou bien d'un film tubulaire dans lequel circulent des ondes visibles [2].

Les sources d'ondes cohérentes, que celles-ci soient de nature élastique ou bien électromagnétique, sont généralement planes; par suite de la symétrie de révolution du guide, nous nous intéresserons également aux sources cylindriques, de même axe que le guide.

Les optiques qui vont être décrites sont destinées au couplage résonnant d'une onde libre émise par une telle source avec une onde guidée dans un tel guide; elles peuvent également servir au couplage d'un guide annulaire avec un récepteur. Il existe principalement deux moyens pour réaliser un tel couplage : les prismes et les réseaux $[3,4]$; nous ne nous intéresserons ici qu'aux prismes, pour lesquels on distingue deux variantes suivant qu'ils sont solides ou liquides.

Durant le couplage d'une source avec un guide, cinq types d'onde sont principalement en présence le long du dioptre qui sépare le coupleur du guide (Fig. 1) : dans le milieu I situé du côté du coupleur, il y a l'onde incidente (1), l'onde réfléchie (2) et l'onde rayonnée par le guide (3); dans le guide, il y a l'onde guidée (4), pour laquelle plusieurs modes sont souvent

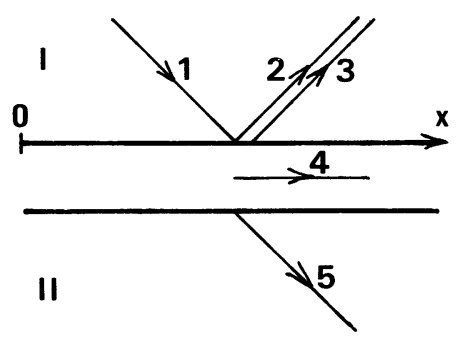

Fig. 1. - Répartition de l'énergie durant le couplage.

[Energy distribution during the coupling.]

possibles; et, dans le milieu II situé de l'autre côté du guide, il peut exister une seconde onde rayonnée (5). Le but du coupleur est d'obtenir le meilleur transfert d'énergie entre l'onde incidente (1) et le mode choisi pour l'onde guidée (4). On peut déjà éliminer l'onde de fuite (5), en choisissant pour le milieu II un indice de réfraction inférieur à celui du guide.

Supposons que l'amplitude de l'onde incidente (1) reste constante le long du dioptre; il en est de même de l'onde réfléchie (2). Au début du couplage, au point $\mathrm{O}$ du dioptre, l'amplitude de l'onde guidée (4) croît proportionnellement à la longueur $x$ du dioptre le long de laquelle le couplage est réalisé $[3,5]$; il en est de même de l'onde rayonnée (3). Cependant, celle-ci est en opposition de phase avec l'onde réfléchie (2) $[5,6]$; il existe donc un point $\mathrm{A}$ du dioptre où le transfert d'énergie atteint $100 \%$, quand les amplitudes des ondes (2) et (3) deviennent égales. Au-delà du point A, l'amplitude de l'onde guidée (4) tend vers un 
maximum qui est atteint en $\mathrm{B}$, lorsque l'énergie qui est soit absorbée ou diffusée dans le guide, soit emportée par l'onde de fuite résultant de l'interférence entre (2) et (3), est égale à l'énergie qui est apportée par (1). $\mathrm{Au}$-delà du point $\mathrm{B}$, elle reste constante si le couplage avec la source est maintenu, elle décroît par absorption et par diffusion si on désaccouple le guide, elle décroît en plus par rayonnement si on désaccouple la source et non le guide.

Lorsque le guide est de révolution, le contact avec la face plane d'un prisme est très local; il en résulte une durée de couplage qui est très brève [2]. C'est pour remédier à cet inconvénient que nous allons chercher à déterminer quels sont les " prismes " qui permettent d'obtenir le couplage le long d'un arc de cercle. Ainsi qu'on le verra, ces nouveaux prismes peuvent même coupler le guide sur $360^{\circ}$, d'où la possibilité de prolonger indéfiniment le couplage; ils ont fait l'objet d'une demande de brevet [7].

2. Principe du calcul. - Soit un faisceau constitué d'ondes cohérentes, dont les surfaces d'onde sont de révolution ; ces ondes peuvent être de nature électromagnétique ou bien encore de nature élastique. Par la suite, nous considérerons comme source (S) l'une quelconque des surfaces d'onde du faisceau. Cette source peut en particulier être cylindrique, de rayon $A$; lorsque ce rayon est très grand, la source est plane et le faisceau est parallèle.

Soit un guide d'onde (G) ayant le même axe de révolution que la source (S). Ce guide doit imposer aux ondes guidées des trajets circulaires de même circonférence, donc de même rayon $R$. Il peut notamment être limité par une surface torique, dont le diamètre du cercle générateur est petit comparé à celui du cercle décrit par le centre du cercle générateur. Il peut aussi être limité par une surface tubulaire, c'està-dire par deux cylindres coaxiaux dont les diamètres sont voisins. Pour que le guide constitue un résonateur, le rayon doit être choisi de façon que la circonférence soit un multiple de la longueur d'onde des ondes guidées.

Nous allons chercher une optique permettant de coupler la source (S) avec le guide (G) le long d'un arc important du guide, pouvant atteindre $360^{\circ}$. Cette optique sera avantageusement constituée d'un seul dioptre (D) agissant par réfraction ou par réflexion; dans ce dernier cas, (D) sera appelé un miroir.

Soit $c_{0}$ la vitesse de phase du faisceau émis par la source (S) et incident sur l'optique (D), $c_{1}$ celle du faisceau émergent de (D) et incident sur le guide (G) et $c_{2}$ celle du mode de propagation des ondes guidées qui nous intéresse dans $(\mathrm{G})$; lorsque l'optique est un miroir, on a bien sûr $c_{0}=c_{1}$. Pour produire des ondes guidées, on doit avoir $c_{1}<c_{2}$.

La condition de couplage entre le faisceau émis et l'onde guidée peut être en particulier exprimée au moyen du principe de Fermat : tous les chemins optiques entre la source (S) et l'une quelconque des sections méridiennes du guide (G) doivent être égaux. $\mathrm{Si}$ nous désignons par $\mathrm{S}$ un point quelconque de (S), par $D$ et par $G$ deux points situés dans le même plan d'incidence que $S$, le premier sur l'optique (D) et le second sur le guide $(G)$, et par $M$ un point donné de (G) situé dans le même plan équatorial du guide que $G$, nous devons avoir :

$$
(\mathrm{SM})=\mathrm{SD} / c_{0}+\mathrm{DG} / c_{1}+\widehat{\mathrm{GM}} / c_{2}=\mathrm{Cte}
$$

ou, en posant $c_{1} / c_{0}=n$ et $c_{1} / c_{2}=\sin i$ :

$$
n \mathrm{SD}+\mathrm{DG}+\widehat{\mathrm{GM}} \sin i=\mathrm{Cte} ;
$$

il suffit cependant que cette condition soit vérifiée avec une tolérance en plus ou en moins égale au quart de la longueur d'onde du faisceau incident sur le guide.

3. Cas d'une source cylindrique. - Considérons une source (S) cylindrique, d'axe $\mathrm{O} z$ et de rayon $A$, et un guide $(\mathrm{G})$ de même axe. Dans cette configuration, il est avantageux de choisir comme optique (D) un dioptre utilisant le phénomène de réfraction. Nous adopterons comme sens de parcours des ondes guidées le sens rétrograde, le guide étant vu de dessus.

Nous supposerons d'abord que le guide $(G)$ est un tore, de centre $\mathrm{O}$. Il sera assimilable à un cercle puisque le rayon du cercle générateur doit être petit comparé au rayon $R$ du cercle décrit par le centre du cercle générateur; sur la figure 2, nous avons pris $A>R$.

Soit $(A, \theta, z)$ les coordonnées cylindriques d'un point quelconque $\mathrm{S}$ de la source $(\mathrm{S})$. Le rayon issu de $\mathrm{S}$ se propage suivant un diamètre de (S) dans le milieu, d'indice relatif $n=c_{1} / c_{0}$, qui est compris entre (S) et l'optique (D); il vient frapper (D) en un point $D$, dont les coordonnées cylindriques sont $(\rho, \theta, z)$, et on a évidemment :

$$
\mathrm{SD}=(-1)^{p}(A-\rho),
$$

avec $p=1$ si $A<R$ ou $p=2 \operatorname{si} A>R$.

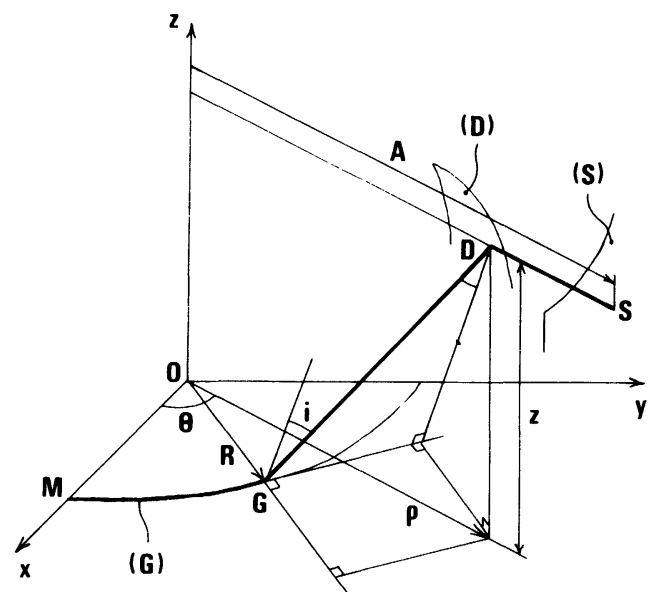

Fig. 2. - Principe du coupleur pour un guide torique.

[Principle of the coupler for a toric guide.] 
Le rayon issu de $\mathrm{S}$ est réfracté par le dioptre (D) suivant un rayon issu de $\mathrm{D}$, qui se propage dans le milieu de couplage où est plongé le guide; l'indice de ce milieu a été pris égal à 1 . Le rayon issu de $D$ est incident sur le tore en un point $\mathrm{G}$ et l'angle d'incidence en ce point doit être tel que $\sin i=c_{1} / c_{2}$. Des relations géométriques simples permettent d'en déduire que la distance $\mathrm{DG}=d$ doit être telle que :

$$
\begin{aligned}
d^{2}+2(-1)^{q} R\left(d^{2} \cos ^{2} i-z^{2}\right)^{1 / 2}+ & \\
& +R^{2}-\rho^{2}-z^{2}=0,
\end{aligned}
$$

avec $q=1$ si $\rho<R$ ou $q=2$ si $\rho>R$.

Dans ces conditions, le rayon issu de $D$ est réfracté en $\mathrm{G}$ de façon à être guidé suivant un grand cercle $\mathrm{de}(\mathrm{G})$ et des relations géométriques simples montrent que :

$$
\widehat{\mathrm{GM}}=R[\theta-\operatorname{Arc} \sin (d \sin i / \rho)] .
$$

En portant dans la condition de couplage (2) les valeurs trouvées respectivement pour SD, DG et $\overline{\mathrm{GM}}$ au moyen des relations (3), (4) et (5), on en tire une équation du dioptre cherché :

$$
\theta=\operatorname{Arcsin}(d \sin i / \rho)+\left[(-1)^{p} n \rho-d\right] / R \sin i,
$$

\section{$d$ étant solution de l'équation (4).}

Supposons maintenant que le guide (G) est un tube d'axe $\mathrm{O} z$. Il sera assimilable à un cylindre puisque l'épaisseur du tube doit être petite comparée au rayon moyen $R$ du tube; sur la figure 3, nous avons pris $A>R$.

Les deux cylindres (S) et $(\mathrm{G})$ sont coaxiaux et les plans d'incidence en sont des plans équatoriaux. Le dioptre cherché (D) est donc un cylindre compris entre $(\mathrm{S})$ et $(\mathrm{G})$ et la figure 3 a été dessinée dans un plan équatorial du guide $(\mathrm{G})$.

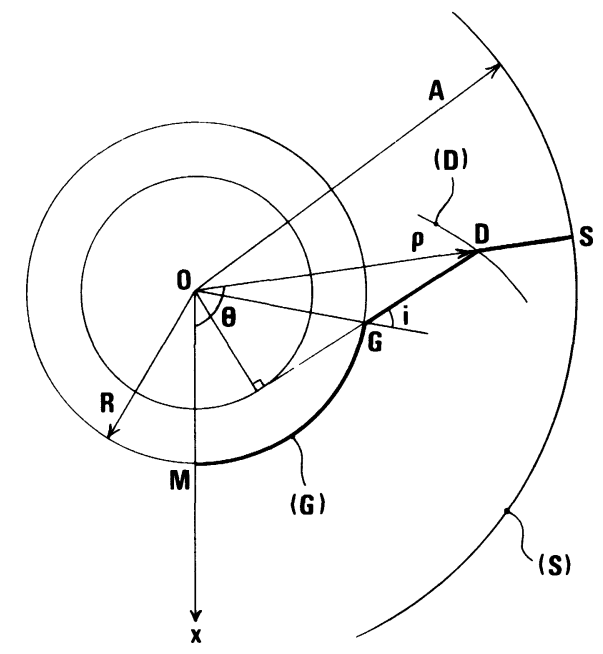

Fig. 3. - Principe du coupleur pour un guide tubulaire.

[Principle of the coupler for a tubular guide.]
Soit $(A, \theta)$ les coordonnées polaires d'un point quelconque $\mathrm{S}$ de la source(S). Le rayon issu de $\mathrm{S}$ se propage suivant un diamètre de (S) dans le milieu, d'indice relatif $n=c_{1} / c_{0}$, qui est compris entre (S) et l'optique (D) ; il vient frapper (D) en un point $D$, dont les coordonnées polaires sont $(\rho, \theta)$, et on a encore la relation (3).

Le rayon issu de $S$ est réfracté par le dioptre (D) suivant un rayon issu de $D$, qui se propage dans le milieu de couplage où est plongé le guide; l'indice de ce milieu a été pris égal à 1 . Le rayon issu de $D$ est incident sur le tube en un point $G$ et l'angle d'incidence en ce point doit être tel que $\sin i=c_{1} / c_{2}$. Des relations géométriques simples permettent d'en déduire que :

$$
\mathrm{DG}=(-1)^{q}\left[\left(\rho^{2}-R^{2} \sin ^{2} i\right)^{1 / 2}-R \cos i\right],
$$

$q$ étant défini comme pour la relation (4).

Dans ces conditions, le rayon issu de $\mathrm{D}$ est réfracté en $\mathrm{G}$ de façon à être guidé suivant un cercle équatorial $\mathrm{de}(\mathrm{G})$ et des relations géométriques simples montrent que :

$$
\widehat{\mathrm{GM}}=(-1)^{q} R[\theta-i+\operatorname{Arc} \sin (R \sin i / \rho)] .
$$

En portant dans la condition de couplage (2) les valeurs trouvées respectivement pour SD, DG et $\overline{\mathrm{GM}}$ au moyen des relations (3), (7) et (8), avec $p=q$ puisque $\rho$ doit être compris entre $A$ et $R$, on en tire une équation d'une section droite de la surface cylindrique du dioptre cherché :

$$
\begin{aligned}
\theta=(-1)^{p} & \{\operatorname{Arc} \cos (R \sin i / \rho)+ \\
+ & {\left.\left[n \rho-\left(\rho^{2}-R^{2} \sin ^{2} i\right)^{1 / 2}\right] / R \sin i\right\} }
\end{aligned}
$$

4. Cas d'une source plane. - Considérons une source (S) plane, perpendiculaire à $\mathrm{O} z$ et située à une distance $B$ de $x \mathrm{O} y$, et un guide (G) d'axe $\mathrm{O} z$. Dans cette configuration, il est avantageux de choisir comme optique (D) un miroir. Nous adopterons le même sens de parcours des ondes guidées qu'au $\S 3$.

Nous supposerons d'abord que le guide $(G)$ est un tore, de centre $\mathrm{O}$, que nous assimilerons encore à un cercle de rayon $R$.

Soit $(\rho, \theta, B)$ les coordonnées cylindriques d'un point quelconque $S$ de la source (S). Le rayon issu de $S$ se propage parallèlement à l'axe $\mathrm{O} z$ dans le milieu où sont à la fois plongés la source (S), le miroir (D) et le guide $(\mathrm{G})$; l'indice relatif de ce milieu a donc été pris égal à 1 . Le rayon issu de $S$ vient frapper (D) en un point $D$, dont les coordonnées cylindriques sont $(\rho, \theta, z)$, et on a évidemment :

$$
\mathrm{SD}=B-(-1)^{r} z,
$$

avec $r=1$ si la source est du côté $z<0$ ou $r=2$ si elle est du côté $z>0$. 
Le rayon issu de $S$ est réfléchi par le miroir (D) suivant un rayon issu de $\mathrm{D}$ et le reste du trajet optique, de $\mathrm{D}$ à $\mathrm{M}$, est identique à celui de la figure 2 . En portant dans la condition de couplage (2) les valeurs trouvées respectivement pour SD, DG et $\widehat{\mathrm{GM}}$ au moyen des relations (10), (4) et (5), on en tire une équation du miroir cherché :

$$
\theta=\operatorname{Arcsin}(d \sin i / \rho)+\left[(-1)^{r} z-d\right] / R \sin i,
$$

$d$ étant solution de l'équation (4).

Supposons maintenant que le guide $(\mathrm{G})$ est un tube, d'axe $\mathrm{O} z$, que nous assimilerons encore à un cylindre de rayon $R$.

$\mathrm{S}$ et $\mathrm{D}$ sont définis comme plus haut pour le guide torique et on a encore la relation (10). Les trois points $\mathrm{D}, \mathrm{G}$ et $\mathrm{M}$ sont dans le même plan équatorial du guide (G) et le reste du trajet optique, de $\mathrm{D}$ à $M$, est identique à celui de la figure 3 . En portant dans la condition de couplage (2) les valeurs trouvées respectivement pour SD, DG et $\widehat{\mathrm{GM}}$ au moyen des relations (10), (7) et (8), on en tire une équation du miroir cherché :

$$
\begin{aligned}
\theta= & (-1)^{q} \operatorname{Arc} \cos (R \sin i / \rho)+ \\
& +\left[(-1)^{r} z-(-1)^{q}\left(\rho^{2}-R^{2} \sin ^{2} i\right)^{1 / 2}\right] / R \sin i .
\end{aligned}
$$

5. Utilisation. - Le fonctionnement de l'optique apparaîtra mieux en considérant un guide tubulaire et un dioptre conforme à l'équation (9); on a vu en effet que les plans d'incidence sont alors des plans équatoriaux. Sur la figure 4 , on a considéré que la résonance dans le guide $(G)$ était obtenue pour une

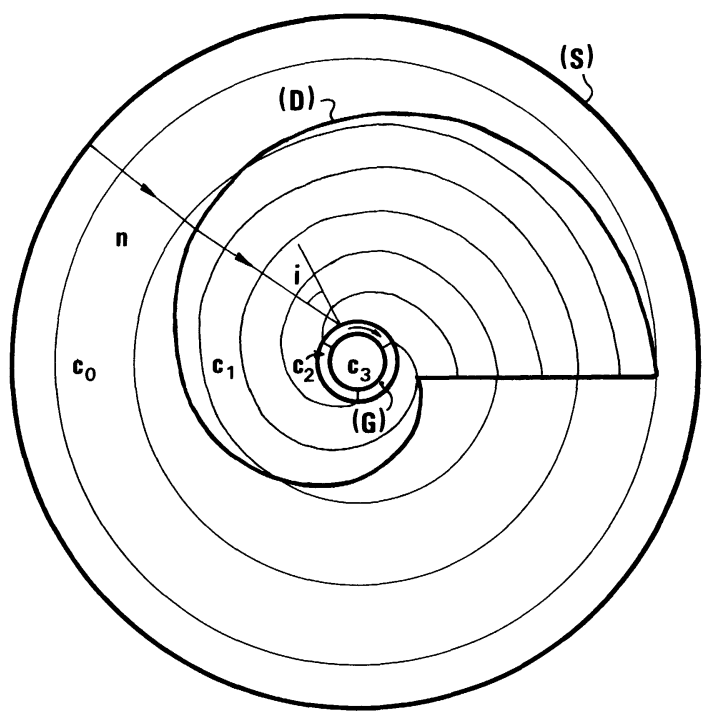

Fig. 4. - Dioptre pour le couplage d'une source cylindrique avec un guide tubulaire.

[Interface for coupling a cylindrical source with a tubular guide.] circonférence égale à 3 longueurs d'onde. Les sections des surfaces d'onde des ondes émises par la source (S) sont circulaires. Après réfraction par le dioptre (D), dont l'indice de réfraction par rapport au milieu de couplage où se trouve le guide est $n=c_{1} / c_{0}=0,5$, les sections des surfaces d'onde se transforment en développantes du cercle de rayon $R \sin i, R$ étant le rayon extérieur du guide tubulaire et $\sin i=c_{1} / c_{2}=$ $0,5\left(i=30^{\circ}\right)$. Ainsi obtient-on la concordance de phase sur $360^{\circ}$ entre les ondes incidentes sur le guide et les ondes guidées. Pour éviter une perte par rayonnement vers l'intérieur du guide, il faut que $c_{2}<c_{3}$.

L'usinage d'un dioptre conforme à l'équation (9) ne pose pas de problème avec une machine à commande numérique. En optique, le guide tubulaire peut être par exemple réalisé par le dépôt d'un film à la surface d'un barreau [8]. Lorsque le "prisme" est solide, l'usinage d'une fente radiale permet d'ajuster par serrage l'épaisseur de la lame d'air au contact du guide.

Déjà en 1973, nous avions proposé un dispositif qui permettait d'obtenir le couplage encerclant d'un guide tubulaire avec une source cylindrique mais non de révolution : la section droite de cette source était en développante de cercle [9], ce qui posait des problèmes de réalisation. C'est pourquoi nous avons ensuite préféré partir d'une source plane et utiliser un miroir conforme à l'équation (12); ce dernier est en effet facilement usinable par fraisage, puisque sa surface est engendrée par la tangente à une hélice circulaire (rayon $a=R \sin i$, pas $=2 \pi a$ ).

Ce miroir a été appliqué à la détection par ultrasons des défauts orientés parallèlement à l'axe dans des tubes minces. En effet, ces défauts constituent des réflecteurs pour des ondes guidées le long des directrices circulaires du tube. Ici, les ondes guidées sont des ondes de Lamb [3]; avec les tubes en zircaloy, qui sont utilisés pour le gainage du combustible des réacteurs nucléaires PWR (diamètre extérieur $2 R=$ $9,5 \mathrm{~mm}$, épaisseur $=0,6 \mathrm{~mm}$ ), c'est le mode $S_{1}$ qui donne les meilleurs résultats. La source ultrasonore est une couronne piézoélectrique, dont la fréquence

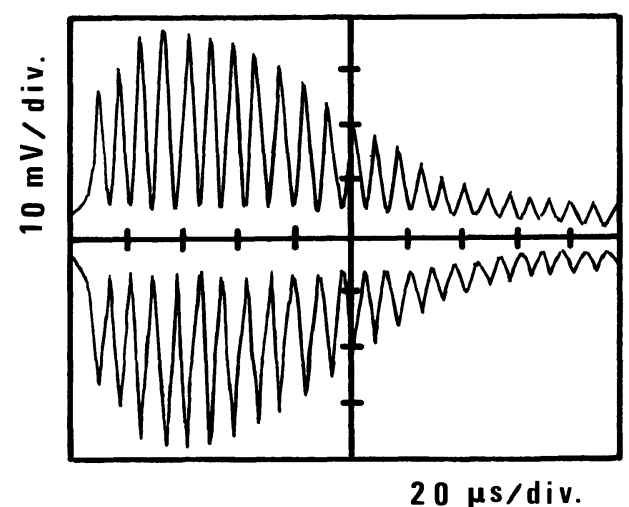

Fig. 5. - Echo multiple dans un tube avec défaut longitudinal.

[Multiple echo in a tube with a longitudinal defect.] 
est de l'ordre de $5 \mathrm{MHz}$ et qui est excitée par des impulsions émises avec une récurrence de l'ordre du $\mathrm{kHz}$, le miroir encerclant est en acier inoxydable $(a=1,4 \mathrm{~mm})$, le milieu situé à l'extérieur du tube est de l'eau et celui situé à l'intérieur est de l'air. Sur l'oscilloscope, on observe en présence d'un défaut une série d'échos équidistants, dont l'amplitude croît avant de décroître lentement avec le retard (Fig. 5); la distance entre deux échos successifs correspond à $30 \mathrm{~mm}$ de zircaloy, soit la circonférence du tube. L'amplitude varie d'environ $6 \mathrm{~dB}$ en 10 tours, alors qu'avec un couplage local l'onde guidée n'arrive pas à effectuer un seul tour. Comme cette résonance est observée quel que soit l'azimut du défaut par rapport au miroir, elle a été exploitée pour réaliser des bancs de contrôle qui ne nécessitent plus la mise en rotation du tube ou de la source [10].

La figure 6 illustre selon deux vues, l'une de face et l'autre de dessus, un exemple de réalisation dans lequel le guide (G) est torique. La source est plane, située parallèlement à $(\mathrm{G})$ au-dessus du miroir $(\mathrm{D})$; celui-ci est conforme à l'équation (11), avec $i=30^{\circ}$.

C'est une nouvelle famille de dioptres et de miroirs qui voit ainsi le jour et nous espérons que, après avoir conduit à une première application en acoustique, elle en trouvera bien d'autres en optique ou en radioélectricité
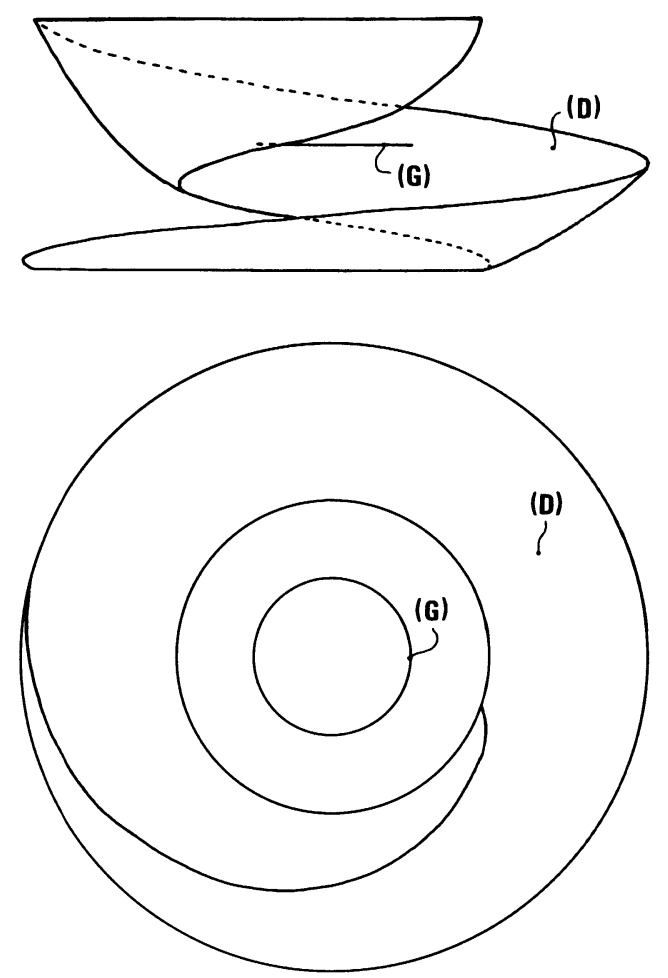

Fig. 6. - Miroir pour le couplage d'une source plane avec un guide torique.

[Mirror for coupling a plane source with a toric guide.]

\section{Bibliographie}

[1] TISCHER, F. J., Resonance properties of ring circuits, IRE Trans. Microwave Theory Tech. MTT 5 (1957) 51.

[2] Weber, H. P. et Ulrich, R., A thin-film ring laser, Appl. Phys. Lett. 19 (1971) 38.

[3] Viktorov, I. A., Rayleigh and Lamb waves (Plenum Press, New York) 1970.

[4] KogelniK, H., Coupling of optical devices and waveguides, dans Photonics, Balkanski, M. et Lallemand, P., éditeurs (Gauthier-Villars, Paris) 1973.

[5] TiEn, P. K. et Ulrich, R., Theory of prism-film coupler and thin-film light guides, J. Opt. Soc. Am. 60 (1970) 1325.
[6] Deighton, M. O., Gillespie, A. B., Pike, R. B. et WATKINS, R. D., Mode conversion of Rayleigh and Lamb waves at a metal-liquid interface, Ultrasonics 19 (1981) 249.

[7] Perdijon, J., Dispositif de couplage encerclant, demande de brevet EN 82 04142, 5 mars 1982.

[8] Ulrich, R. et Weber, H. P., Solution-deposited thin films as passive and active light-guides, Appl. Opt. 11 (1972) 428.

[9] Perdijon, J., Traducteur ultrasonore à caustique cylindrique ou sphérique, Revue Phys. Appl. 9 (1974) 925.

[10] Perdijon, J., Nondestructive testing of tubes by encircling sensors, Mater. Eval. 39 (1981) 1222. 